\title{
Data-Driven Minimum-Energy Controls for Linear Systems
}

\author{
Giacomo Baggio, Vaibhav Katewa, and Fabio Pasqualetti
}

\begin{abstract}
In this paper we study the problem of computing minimum-energy controls for linear systems from experimental data. The design of open-loop minimum-energy control inputs to steer a linear system between two different states in finite time is a classic problem in control theory, whose solution can be computed in closed form using the system matrices and its controllability Gramian. Yet, the computation of these inputs is known to be ill-conditioned, especially when the system is large, the control horizon long, and the system model uncertain. Due to these limitations, open-loop minimum-energy controls and the associated state trajectories have remained primarily of theoretical value. Surprisingly, in this paper we show that open-loop minimum-energy controls can be learned exactly from experimental data, with a finite number of control experiments over the same time horizon, without knowledge or estimation of the system model, and with an algorithm that is significantly more reliable than the direct model-based computation. These findings promote a new philosophy of controlling large, uncertain, linear systems where data is abundantly available.
\end{abstract}

Index Terms - Linear systems, optimal control, statistical learning, identification for control, control of networks.

\section{INTRODUCTION}

$\mathbf{C}$ ONSIDER the discrete-time linear time-invariant system

$$
x(t+1)=A x(t)+B u(t),
$$

where, respectively, $A \in \mathbb{R}^{n \times n}$ and $B \in \mathbb{R}^{n \times m}$ denote the system and input matrices, and $x: \mathbb{N} \rightarrow \mathbb{R}^{n}$ and $u: \mathbb{N} \rightarrow \mathbb{R}^{m}$ describe the state and input of the system. For a control horizon $T \in \mathbb{N}$ and a desired state $x_{\mathrm{f}}$, the minimum-energy control problem asks for the input sequence $u(0), \ldots, u(T-1)$ with minimum energy that steers the state from $x_{0}$ to $x_{\mathrm{f}}$ in $T$ steps, and it can be formulated as

$$
\begin{array}{ll}
\min _{u} & \sum_{t=0}^{T-1}\|u(t)\|_{2}^{2}, \\
\text { s.t. } & x(t+1)=A x(t)+B u(t), \\
& x(0)=x_{0}, x(T)=x_{\mathrm{f}} .
\end{array}
$$

As a classic result [1], the minimization problem (2]) is feasible if and only if $\left(x_{\mathrm{f}}-A^{T} x_{0}\right) \in \operatorname{Im}\left(W_{T}\right)$, where

$$
W_{T}=\sum_{t=0}^{T-1} A^{t} B B^{\top}\left(A^{\top}\right)^{t}
$$

This material is based upon work supported in part by ARO 71603NSYIP. Giacomo Baggio, Vaibhav Katewa and Fabio Pasqualetti are with the Department of Mechanical Engineering, University of California at Riverside, $\{$ gbaggio vkatewa. fabiopas\}eengr.ucr.edu. is the $T$-steps controllability Gramian and $\operatorname{Im}\left(W_{T}\right)$ denotes the image of the matrix $W_{T}$. Further, the solution to (2) is

$$
u^{*}(t)=B^{\top}\left(A^{\top}\right)^{T-t-1} W_{T}^{\dagger}\left(x_{\mathrm{f}}-A^{T} x_{0}\right),
$$

where $W_{T}^{\dagger}$ is the Moore-Penrose pseudoinverse of $W_{T}$ [2].

The controllability Gramian (3) and the minimum-energy control input (4) identify fundamental control limitations for the system (1), and have been extensively used to solve design [3], sensor and actuator placement [4], and control problems [5] for systems and networks. However, besides their theoretical value, the optimal control input (4) is rarely used in practice or even computed numerically because (i) it relies on the perfect knowledge of the system dynamics, (ii) its performance is not robust to model uncertainties, and (iii) the controllability Gramian is typically ill-conditioned, especially when the system is large [5], [6]. This implies that the control sequence (4) is numerically difficult to compute, and that its implementation leads to errors [7]. To the best of our knowledge, efficient and numerically reliable methods to compute minimum-energy control inputs are still lacking.

Paper contributions. This paper features two main contributions. First, we show that minimum-energy control inputs for linear systems can be computed from data obtained from control experiments with non-minimum-energy inputs, and without knowledge or estimation of the system matrices. Thus, optimal inputs can be learned from non-optimal ones, and we provide three different expressions for doing so. Surprisingly, we also establish that a finite number of non-optimal control experiments is always sufficient to compute minimum-energy control inputs towards any reachable state. Second, we show that the data-driven computation of minimum-energy inputs is numerically as reliable as the computation of the inputs based on the exact knowledge of the system matrices, and substantially more reliable than using the closed-form expression based on the Gramian. Further, as minor contributions, we (i) derive bounds on the number of required control experiments as a function of the dimension of the system, number of control inputs, and length of the control horizon, (ii) discuss the effect of noisy data on the data-driven expressions, and (iii) extend our data-driven framework to the case of output measurements.

Our results suggest the tantalizing hypothesis that several optimal control problems can be solved efficiently and reliably using a combination of data-driven algorithms and system properties (in our setup, linearity of the dynamics), even when the system model is uncertain or unknown.

Related work. Several works investigate the problem of estimating optimal controls for linear systems from inputoutput data. The classic model-based approach [8] consists of 
(i) identifying a model of the system from the available data, and (ii) using the estimated model to design the optimal control inputs. Data-driven algorithms have been proposed in [9][12] for the LQR/LQG problem. In particular, the approach pursued in these papers relies on the estimation of the Markov parameters of the system, thereby bypassing the identification step of the model-based approach. Differently from the above approaches, in this paper we focus on computing openloop minimum-energy inputs from experimental data, without reconstructing the system matrices and where the experiments use arbitrary control inputs. To the best of our knowledge, this paper addresses a novel problem and provides new and numerically more reliable expressions for the computation of minimum-energy control inputs.

\section{LEARNING MINIMUM-ENERGY CONTROL INPUTS}

In vector form, the minimum-energy control problem asks to find the minimum-norm solution to the following equation:

$$
x_{\mathrm{f}}=A^{T} x_{0}+\underbrace{\left[\begin{array}{llll}
B & A B & \cdots & A^{T-1} B
\end{array}\right]}_{C_{T}} u,
$$

where the vector $u \in \mathbb{R}^{m T}$ contains the control inputs over the control horizon $[0, T-1]$, namely $u=\left[u(T-1)^{\top} \cdots u(0)^{\top}\right]^{\top}$, and $C_{T}$ denotes the $T$-steps controllability matrix 1 Then, if the controllability matrix $C_{T}$ is known, the minimum-energy control input to reach $x_{\mathrm{f}}$ is

$$
u^{*}=C_{T}^{\dagger}\left(x_{\mathrm{f}}-A^{T} x_{0}\right) .
$$

Instead of using (5), in this paper we aim to compute minimum-energy control inputs leveraging a set of $N$ control experiments and assuming that the system matrices, and thus the controllability matrix, are not available. The $i$-th control experiment consists of applying the input sequence $u_{i}$ to (1), and measuring the system state at time $T$, namely $x_{i}$, where

$$
x_{i}=A^{T} x_{0}+C_{T} u_{i}
$$

We remark that the inputs $u_{i}$ are arbitrary and not necessarily of minimum-norm. In vector form, the available data is

$$
X=\left[\begin{array}{lll}
x_{1} & \cdots & x_{N}
\end{array}\right], \text { and } U=\left[\begin{array}{lll}
u_{1} & \cdots & u_{N}
\end{array}\right],
$$

where $x_{i}$ is the state at time $T$ with input $u_{i}$ as in (6) $2^{2}$

\section{A. Data-driven minimum-energy controls}

Because we only rely on the experimental data $(X, U)$ to learn the minimum-energy control input to reach a desired state, we postulate that such input can be computed as a linear combination of the inputs $U$. Thus, we formulate and study the following constrained minimization problem:

$$
\begin{array}{cc}
\alpha^{*}=\arg \min _{\alpha} & \|U \alpha\|_{2}^{2}, \\
\text { s.t. } & x_{\mathrm{f}}=X \alpha,
\end{array}
$$

\footnotetext{
${ }^{1}$ To simplify the technical treatment and without compromising generality, we assume that $x_{\mathrm{f}}$ is reachable in $T$-steps, i.e., $\left(x_{\mathrm{f}}-A^{T} x_{0}\right) \in \operatorname{Im}\left(C_{T}\right)$.

${ }^{2}$ While the full state trajectory could be measured [13], here we show that measuring the final state is sufficient to compute minimum-energy inputs.
}

where $\alpha \in \mathbb{R}^{N}$ is the optimization variable. As we show in Theorem 2.1, a first data-driven expression for the minimumenergy control input derives from a solution to (8). We start with the expression of the minimum-energy control input for the case $x_{0}=0$, and we postpone the general case $x_{0} \neq 0$ to Remark 2 Let $\operatorname{Im}(M)$ and $\operatorname{Ker}(M)$ denote the range-space and the null-space of the matrix $M$, respectively. With a slight abuse of notation, we write $K=\operatorname{Im}(A)($ resp. $K=\operatorname{Ker}(A)$ ) to say that $K$ is a basis of $\operatorname{Im}(A)$ (resp. $\operatorname{Ker}(A))$. A matrix is full row rank if the dimension of its range-space equals the number of its rows.

Theorem 2.1: (Data-driven minimum-energy control inputs when $x_{0}=0$ ) If the matrix $U$ in (7) is full row rank, then, for any final state $x_{\mathrm{f}}$, the minimum-energy input equals

$$
u^{*}=\left(I-U K(U K)^{\dagger}\right) U X^{\dagger} x_{\mathrm{f}},
$$

where $K=\operatorname{Ker}(X)$ and $X$ is as in (7).

Proof: We first show that $(8)$ is feasible, and that $u^{*}=$ $U \alpha^{*}$. Notice that, because $U$ is full row rank, there exists $\alpha^{*}$ such that $u^{*}=U \alpha^{*}$, where $u^{*}$ is the minimum-energy control input to reach $x_{\mathrm{f}}$. Additionally, $\alpha^{*}$ satisfies the constraint in (8) because $X \alpha^{*}=C_{T} U \alpha^{*}=C_{T} u^{*}=x_{\mathrm{f}}$. Finally, because $u^{*}$ is unique [1], $\alpha^{*}$ is also a solution to (8), and its computation is equivalent to computing the input $u^{*}$.

To compute $\alpha^{*}$ we solve the constraint $x_{\mathrm{f}}=X \alpha$ and substitute it in the cost function. Namely, $\alpha=X^{\dagger} x_{\mathrm{f}}-K w$, where $K=\operatorname{Ker}(X)$ and $w$ is an arbitrary vector. Equating to zero the derivative of the cost function with respect to $w$, we obtain $w^{*}=(U K)^{\dagger} U X^{\dagger} x_{\mathrm{f}}$. This implies that $\alpha^{*}=X^{\dagger} x_{\mathrm{f}}-K w^{*}$, from which $(9)$ follows by letting $u^{*}=U \alpha^{*}$.

Theorem 2.1 provides an expression of the minimum-energy control input, which only uses data originated from a set of control experiments, and does not require the knowledge of the system matrices. Importantly, Theorem 2.1 shows that minimum-energy control inputs can be directly computed based on a number of control experiments with arbitrary, thus not minimum-energy, inputs. Further, Theorem 2.1 assumes that $U$ is full row rank, which guarantees the computation of the minimum-energy input for any final state $x_{\mathrm{f}}$. When $U$ is not full row rank but $u^{*} \in \operatorname{Im}(U)$, the minimum-energy control input can still be computed as in Theorem 2.1. Instead, when $u^{*} \notin \operatorname{Im}(U)$, the minimum-energy input cannot be computed as a (linear) combination of the experimental data (7). In this case, the data-driven input (9) reaches the desired final state $x_{\mathrm{f}}$, if $x_{\mathrm{f}} \in \operatorname{Im}(X)$, or the final state $\tilde{x}_{\mathrm{f}} \in \operatorname{Im}(X)$ that is closest to $x_{\mathrm{f}}$, if $x_{\mathrm{f}} \notin \operatorname{Im}(X)$. To see this, let $u^{*}$ be as in 9) and note that

$$
\begin{aligned}
\tilde{x}_{\mathrm{f}} & =C_{T} u^{*}=C_{T}\left(I-U K(U K)^{\dagger}\right) U X^{\dagger} x_{\mathrm{f}} \\
& =C_{T} U X^{\dagger} x_{\mathrm{f}}-\underbrace{C_{T} U K(U K)^{\dagger} U X^{\dagger} x_{\mathrm{f}}}_{=0 \text { because } C_{T} U K=X K=0}=X X^{\dagger} x_{\mathrm{f}},
\end{aligned}
$$

which shows that $\tilde{x}_{\mathrm{f}}$ is the orthogonal projection of $x_{\mathrm{f}}$ onto $\operatorname{Im}(X)$. This in particular implies that the error $\left\|x_{\mathrm{f}}-\tilde{x}_{\mathrm{f}}\right\|_{2}$ is non-increasing in the number of experiments $N$, and it vanishes when the experimental data satisfies $x_{\mathrm{f}} \in \operatorname{Im}(X)$. Finally, Theorem 2.1 can also be used to quantify the number of experiments needed to compute minimum-energy inputs.

Corollary 2.2: (Required number of control experiments to compute minimum-energy inputs) Let $n$ be the dimension 


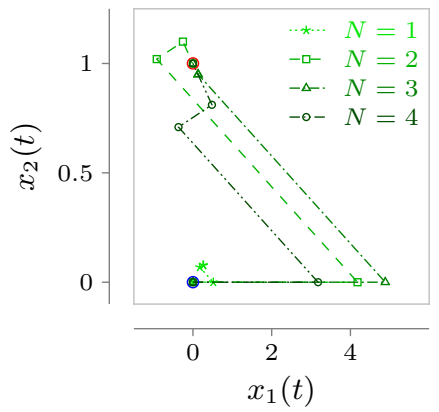

(a)
$A=\left[\begin{array}{rl}-0.5 & 0 \\ 0.1 & 0.8\end{array}\right] \quad B=\left[\begin{array}{l}1 \\ 0\end{array}\right]$

(b)

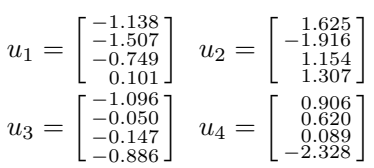

(c)

$\left\|u^{*}\right\|_{2}=9.317$

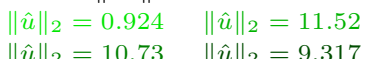

(d)
Fig. 1. Fig. 1 a) illustrates the state trajectory $x(t)=\left[x_{1}(t) x_{2}(t)\right]^{\top}, t \in$ $[0, T]$, of the system described by matrices $A, B$ as in Fig. 1 b) and driven by the data-driven input $\hat{u}(9$, for four values of the number of experiments $N$. We choose $T=4$ and denote the initial state $x_{0}=\left[\begin{array}{ll}0 & 0\end{array}\right]^{\top}$ and final state $x_{\mathrm{f}}=\left[\begin{array}{ll}0 & 1\end{array}\right]^{\top}$ with a blue and red circle, respectively. The other markers correspond to the values of the trajectories in the interval $[0, T]$. The datadriven input $\hat{u}$ has been computed using input data as in $u_{i}$ as Fig. 1. c), and $x_{i}=C_{T} u_{i}$. Fig. 1]d) shows the norm of the minimum-energy input and the norm of the data-driven input $\hat{u}$ (9) as $N$ varies (color-coded).

of the system, $m$ the number of inputs, $T$ the control horizon, and $N$ the number of control experiments. Then,

(i) $N \geq n$ is necessary to compute minimum-energy control inputs towards any arbitrary final state $x_{\mathrm{f}}$;

(ii) $N=m T$ is sufficient to compute minimum-energy control inputs towards any arbitrary final state $x_{\mathrm{f}}$, provided that the inputs $u_{i}$ are linearly independent.

Proof: (Necessity) Assume by contradiction that the number of experiments is strictly less than $n$. Then, $\operatorname{Rank}(X)<n$, and there exists $x_{\mathrm{f}} \notin \operatorname{Im}(X)$. Then, the minimization problem (8) is infeasible, and the minimum-energy control input cannot be computed from the inputs $U$.

(Sufficiency) Let the experimental inputs be linearly independent. Then, $U$ is invertible and, for any $x_{\mathrm{f}}$, there exists a solution $\alpha^{*}$ such that $u^{*}=U \alpha^{*}$. This shows that the minimum-energy input can be computed from the data.

Corollary 2.2 characterizes the number of control experiments that are required to compute minimum-energy control inputs from experimental data. In particular, as few as $n$ experiments are needed, in which case the experiments must contain $n$ linearly independent minimum-energy control inputs, and as many as $m T$ experiments are sufficient, in which case the control inputs can be selected arbitrarily provided that they form a linearly independent set of vectors. This also shows that optimal control inputs can be learned from a finite number of non-optimal control inputs.

Example 1: (Data-driven control inputs when $N \leq m T$ ) We consider a two-dimensional system with matrices $A$ and $B$ as in Fig. 1 (b), control horizon $T=4$, initial state $x_{0}=\left[\begin{array}{ll}0 & 0\end{array}\right]^{\top}$, and final state $x_{\mathrm{f}}=\left[\begin{array}{ll}0 & 1\end{array}\right]^{\top}$. We vary the number of control experiments $N$ from 1 to 4 , where the inputs are as in Fig. 1. (c). For each number of control experiments, we compute the datadriven input (9), and report the corresponding state trajectory and norm in Fig. 1(a) and Fig. 1(d), respectively. Notice that, when $N=1$, the data-driven input does not steer the system state to $x_{\mathrm{f}}$. Instead, for $N=2,3,4$ the state trajectory reaches $x_{\mathrm{f}}$. Finally, the data-driven input has minimum norm only when $N=4$.

Remark 1: (Geometric properties of (9)) Several geometric properties of (9) can be highlighted. First, $U K=\operatorname{Ker}\left(C_{T}\right)$ when $U$ is full row rank. In fact, $C_{T} U K=X K=0$, showing that $\operatorname{Im}(U K) \subseteq \operatorname{Ker}\left(C_{T}\right)$. Further, if $C_{T} u=0$ and $u=U \alpha$, then, $X \alpha=C_{T} U \alpha=C_{T} u=0$, showing that $\alpha \in \operatorname{Im}(K)$ and $\operatorname{Ker}\left(C_{T}\right) \subseteq \operatorname{Im}(U K)$. Thus, $\operatorname{Im}(U K)=\operatorname{Ker}\left(C_{T}\right)$ when $U$ is full row rank. Second, $I-U K(U K)^{\dagger}$ is the orthogonal projection onto the kernel of $(U K)^{\top}$ and, consequently, $u^{*}=\left(I-U K(U K)^{\dagger}\right) U X^{\dagger} x_{\mathrm{f}}$ is orthogonal to $\operatorname{Ker}\left(C_{T}\right)$. This is expected, because $u^{*}$ is the minimum-energy control input to reach the state $x_{\mathrm{f}}$.

\section{B. An alternative expression of minimum-energy controls}

In this subsection, we present a different optimization problem that can be used to derive an equivalent expression of the data-driven minimum-energy control input 9 . Specifically, we consider the following problem, which encodes the problem of estimating the controllability matrix from data:

$$
C_{T}^{*}=\arg \min _{C}\|X-C U\|_{F}^{2},
$$

where $\|\cdot\|_{F}$ denotes the Frobenius norm of a matrix. The above problem has a unique solution, which equals $C_{T}^{*}=X U^{\dagger}$. Notice that the minimization problem (10) returns an estimate of the controllability matrix, which can be used to compute the input as $\hat{u}=\left(C_{T}^{*}\right)^{\dagger} x_{\mathrm{f}}=\left(X U^{\dagger}\right)^{\dagger} x_{\mathrm{f}}$. We next show that $\hat{u}$ coincides with the control input $(9)$.

Theorem 2.3: (Equivalent expressions of data-driven minimum-energy inputs) Let $X$ and $U$ be as in (7). Then,

$$
\left(I-U K(U K)^{\dagger}\right) U X^{\dagger} x_{\mathrm{f}}=\left(X U^{\dagger}\right)^{\dagger} x_{\mathrm{f}} .
$$

Proof: We show that $\left(X U^{\dagger}\right)^{\dagger}=\left(I-U K(U K)^{\dagger}\right) U X^{\dagger}$. That is, we show that $\left(I-U K(U K)^{\dagger}\right) U X^{\dagger}$ satisfies the four conditions [2] defining the Moore-Penrose pseudoinverse of $X U^{\dagger}$. To this aim, let $K=I-X^{\dagger} X$. Since $P=$ $I-U K(U K)^{\dagger}$ is the orthogonal projection onto $\operatorname{Ker}\left((U K)^{\top}\right)$,

$$
(U K)^{\top} P=0 \stackrel{P=P^{\top}}{\Longrightarrow} P U K=0 \Rightarrow P U X^{\dagger} X=P U .
$$

Because $X=C_{T} U$, we have $\operatorname{Ker}(U) \subseteq \operatorname{Ker}(X)$. Since $I-$ $U^{\dagger} U$ is the orthogonal projection onto $\operatorname{Ker}(U)$, we have

$$
X\left(I-U^{\dagger} U\right)=0 \Rightarrow X U^{\dagger} U=X .
$$

Further, using $X K=0$, we obtain

$$
X U^{\dagger}(I-P)=X U^{\dagger} U K(U K)^{\dagger} \stackrel{13}{=} X K(U K)^{\dagger}=0 .
$$

Finally, since $I-U U^{\dagger}$ denotes the orthogonal projection onto $\operatorname{Ker}\left(U^{\top}\right)$, and $U K(U K)^{\dagger}$ the orthogonal projection onto $\operatorname{Im}(U K) \subseteq \operatorname{Im}(U) \perp \operatorname{Ker}\left(U^{\top}\right)$, we have

$$
\begin{aligned}
& U K(U K)^{\dagger}=I-P=0 \\
\Rightarrow & (I-P)\left(I-U U^{\dagger}\right)=\left[(I-P)\left(I-U U^{\dagger}\right)\right]^{\top} \\
\Rightarrow & U U^{\dagger} P=P U U^{\dagger},
\end{aligned}
$$

where the last implication follows because $I-P$ and $I-U U^{\dagger}$ are symmetric. To conclude, we show that $P U X^{\dagger}=\left(X U^{\dagger}\right)^{\dagger}$ by proving the four Moore-Penrose conditions [2]: 

(i) $P U X^{\dagger} X U^{\dagger} P U X^{\dagger} \stackrel{12}{=} P U U^{\dagger} P U X^{\dagger} \stackrel{15}{=} P^{2} U U^{\dagger}$. $U X^{\dagger}=P U X^{\dagger}$
(ii) $X U^{\dagger} P U X^{\dagger} X U^{\dagger} X U^{\dagger} P U U^{\dagger}=X U^{\dagger} U U^{\dagger}-$ $X U^{\dagger}(I-P) U U^{\dagger} \stackrel{14}{=} X U^{\dagger}$
(iii) $X U^{\dagger} P U X^{\dagger}=X U^{\dagger} U X^{\dagger}-X U^{\dagger}(I-P) U X^{\dagger}$ $X X^{\dagger}=\left(X X^{\dagger}\right)^{\top}$
(iv) $P U X^{\dagger} X U^{\dagger} \stackrel{[12}{=} P U U^{\dagger} \stackrel{[15}{=} U U^{\dagger} P=\left(P U U^{\dagger}\right)^{\top}$.

\section{An asymptotic expression of minimum-energy controls}

The minimization problem (10) reconstructs the forward controllability matrix $C_{T}$, from which minimum-energy control inputs can be derived by subsequently computing $C_{T}^{\dagger}$. To avoid the computation of $C_{T}^{\dagger}$ and obtain a potentially simpler expression, we next consider the problem of directly estimating $C_{T}^{\dagger}$ from the experimental data:

$$
M^{*}=\arg \min _{M}\|M X-U\|_{F}^{2} .
$$

Notice that the latter problem is equivalent to estimating the inverse map from $X$ to $U$, and it is typically more difficult than the problem of estimating the map from $U$ to $X$. In fact, while the forward map is unique, the inverse map is typically not ${ }^{3}$ Further, the control input $M^{*} x_{\mathrm{f}}$ obtained by solving the minimization problem (16) is not guaranteed to be of minimum norm and to steer the system to $x_{\mathrm{f}}$, as these constraints do not appear in the minimization problem. In what follows, we say that a sequence of random matrices $\left\{X_{n}\right\}_{n \in \mathbb{N}}$ converges almost surely (a.s.) to a matrix $X$, and denote it with $X_{n} \stackrel{\text { a.s. }}{\longrightarrow}$ $X$, if $\operatorname{Pr}\left(\lim _{n \rightarrow \infty} X_{n}=X\right)=1$.

Theorem 2.4: (Asymptotically equivalent expression to 9p) Let $X$ and $U$ be as in (7). The unique solution to the minimization problem 16 is

$$
M^{*}=U X^{\dagger},
$$

and the corresponding control input can be written as

$$
\hat{u}=M^{*} x_{\mathrm{f}}=U X^{\dagger} x_{\mathrm{f}} .
$$

Further, if $X$ is full row rank, then $C_{T} M^{*} x_{\mathrm{f}}=x_{\mathrm{f}}$. That is, the control $\hat{u}$ steers the system from $x_{0}=0$ to $x(T)=x_{\mathrm{f}}$. Finally, if the entries of $U$ are i.i.d. random variables with zero mean and nonzero finite variance, then $U X^{\dagger} \stackrel{\text { a.s. }}{\longrightarrow} C_{T}^{\dagger}$ as $N \rightarrow \infty$. That is, as the number of control experiments increases, the input $\hat{u}$ converges a.s. to the optimal input $u^{*}$.

Proof: The expression (17) follows from the properties of the Moore-Penrose pseudoinverse. For the second claim, we note that $C_{T} \hat{u}=C_{T} U X^{\dagger} x_{\mathrm{f}}=X X^{\dagger} x_{\mathrm{f}}=x_{\mathrm{f}}$, where we have used that $X$ is full row rank and $X=C_{T} U$. To prove the third statement, let $N \rightarrow \infty$, and let the control experiments be chosen so that the entries of $U$ are i.i.d. random variables with zero mean and finite variance $\sigma^{2}$. Let $U_{i j}$ denote the $(i, j)$-th entry of $U$, and observe that the $(i, j)$-th entry of $\frac{1}{N} U U^{\top}$ equals $\frac{1}{N} \sum_{k=1}^{N} U_{i k} U_{j k}$. Because $\left\{U_{i k} U_{j k}\right\}_{k \in \mathbb{N}}$ is an i.i.d. sequence of random variables, for all $i, j \in\{1, \ldots, N\}$

\footnotetext{
${ }^{3}$ In particular, the inverse map is not unique whenever $m T>n$.
}

and, due to the Strong Law of Large Numbers [14, p. 6], when $N \rightarrow \infty$ we have

$$
\frac{1}{N} \sum_{k=1}^{N} U_{i k} U_{j k} \stackrel{\text { a.s. }}{\longrightarrow} \mathbb{E}\left[U_{i 1} U_{j 1}\right]= \begin{cases}\sigma^{2}, & \text { if } i=j, \\ 0, & \text { if } i \neq j,\end{cases}
$$

where $\mathbb{E}[\cdot]$ denotes the expected value operator. Then,

$$
\frac{1}{N} U U^{\top} \stackrel{\text { a.s. }}{\longrightarrow} \sigma^{2} I \quad \text { as } N \rightarrow \infty \text {. }
$$

Next, consider the function $f: \mathbb{R}^{m T \times m T} \rightarrow \mathbb{R}^{m T \times n}, Y \mapsto$ $Y C_{T}^{\mathrm{T}}\left(C_{T} Y C_{T}^{\mathrm{T}}\right)^{\dagger}$. Note that $f(Y)$ is continuous at $Y=\alpha I$, $\alpha>0.4$ and $f(\alpha I)=C_{T}^{\top}\left(C_{T} C_{T}^{\top}\right)^{\dagger}=C_{T}^{\dagger}$ [2, p. 49]. To conclude, we employ the Continuous Mapping Theorem [14. Theorem 2.3(iii)] and (19) to obtain, as $N \rightarrow \infty$,

$$
\begin{aligned}
U X^{\dagger} & =U\left(C_{T} U\right)^{\dagger}=\frac{1}{N} U U^{\top} C_{T}^{\top}\left(C_{T} \frac{1}{N} U U^{\top} C_{T}^{\top}\right)^{\dagger} \\
& =f\left(\frac{1}{N} U U^{\top}\right) \stackrel{\text { a.s. }}{\longrightarrow} f\left(\sigma^{2} I\right)=C_{T}^{\dagger} .
\end{aligned}
$$

Theorem 2.4 contains a data-driven expression of the minimum-energy control input for a linear system, which does not rely on the estimation of the system matrices or the controllability matrix. As we show in the next section, the expression (18) is not only conceptually simpler than the classic Gramian-based expression of the minimum-energy control input and our other data-driven expressions $(9)$ and (11), but it is also numerically more reliable as it requires a smaller number of operations. Yet, differently from $(9)$ and (11), the expression (18) coincides with the minimum-energy control only asymptotically in the number of experiments, and assuming that the entries of the input matrix $U$ are zero-mean i.i.d. random variables with nonzero finite variance.

Remark 2: (Data-driven minimum-energy control inputs when $x_{0} \neq 0$ ) When $x_{0} \neq 0$, the computation of the minimum-energy control input to reach $x_{\mathrm{f}}$ is more involved, as the unknown matrix $A$ and vector $x_{0}$ enter the relation (6) 5 Yet, under a mild assumption on the experimental inputs $U$, minimum-energy inputs can still be computed with a finite number of experiments. To see this, consider the problem

$$
\begin{array}{ll}
\min _{\alpha} & \|U \alpha\|_{2}^{2}, \\
\text { s.t. } & x_{\mathrm{f}}=X \alpha \text { and } 1=\mathbb{1}^{\top} \alpha,
\end{array}
$$

Assume that the matrix $U$ is full row rank, and that there exists a vector $w$ such that $U w=0$ and $\mathbb{1}^{\top} w \neq 0$. The first assumption guarantees that there exists $\alpha^{*}$ such that $u^{*}=$ $U \alpha^{*}$, and thus the computation of the minimum-energy control for any final state $x_{\mathrm{f}}$ (cf. Theorem 2.1). The second assumption ensures that there exists $\alpha^{*}$ satisfying $1=\mathbb{1}^{\top} \alpha^{*}$, which allows us to correctly reconstruct the term $A^{T} x_{0}$ from $X{ }^{6}$ In fact,

\footnotetext{
${ }^{4}$ In fact, since $\operatorname{Rank}\left(C_{T} Y C_{T}^{\mathrm{T}}\right)=\operatorname{Rank}\left(C_{T} C_{T}^{\mathrm{T}}\right)$ for any positive definite $Y$, it holds $\lim _{k \rightarrow \infty}\left(C_{T} Y_{k} C_{T}^{\top}\right)^{\dagger}=\left(\alpha C_{T} C_{T}^{\top}\right)^{\dagger}$ for any sequence of positive definite matrices $\left\{Y_{k}\right\}_{k \in \mathbb{N}}$ such that $\lim _{k \rightarrow \infty} Y_{k}=\alpha I$ [2] p. 238].

${ }^{5}$ Notice that the term $A^{T} x_{0}$ remains unknown even if the exact value of $x_{0} \neq 0$ is known. Thus knowledge of $x_{0}$ does not modify the expressions we obtain when $x_{0} \neq 0$ is treated as an unknown variable.

${ }^{6}$ These assumptions can always be satisfied by properly designing the experimental inputs, or by running sufficiently many random experiments.
} 
let $\alpha^{*}=U^{\dagger} u^{*}+w\left(1-\mathbb{1}^{\top} U^{\dagger} u^{*}\right) /\left(\mathbb{1}^{\top} w\right)$, and notice that $u^{*}=U \alpha^{*}$, where $u^{*}$ is the minimum-energy control input to reach $x_{\mathrm{f}}$. Further, using (6) and $1=\mathbb{1}^{\top} \alpha^{*}$, we have $X \alpha^{*}=$ $\sum_{i=1}^{N} X_{i} \alpha_{i}^{*}=A^{T} x_{0} \sum_{i=1}^{N} \alpha_{i}^{*}+C_{T} \sum_{i=1}^{N} \alpha_{i}^{*} U_{i}=A^{T} x_{0}+$ $C_{T} u^{*}=x_{\mathrm{f}}$. Then, similarly to the proof of Theorem 2.1. a solution to 20 determines the minimum-energy input.

To solve the minimization problem $(20)$, let $\bar{X}=\left[X^{\top} \mathbb{1}\right]^{\top}$ and $\bar{x}_{\mathrm{f}}=\left[\begin{array}{ll}x_{\mathrm{f}}^{\top} & 1\end{array}\right]^{\top}$. Then, similarly to Theorem 2.1, we obtain $\alpha^{*}=\bar{X}^{\dagger} x_{\mathrm{f}}-K(U K)^{\dagger} U \bar{X}^{\dagger} \bar{x}_{\mathrm{f}}$, where $K=\operatorname{Ker}(X)$, and

$$
u^{*}=\left(I-U K(U K)^{\dagger}\right) U \bar{X}^{\dagger} \bar{x}_{\mathrm{f}} .
$$

Because the matrix $U$ is required to have a nontrivial nullspace, a sufficient number of linearly-independent non-optimal experiments for the computation of the minimum-energy control input to any arbitrary final state is $m T+1$.

Finally, from the above reasoning and the proof of Theorem 2.3 and Theorem 2.4, the minimum-energy input (21) can be written equivalently as $u^{*}=\left(\bar{X} U^{\dagger}\right)^{\dagger} \bar{x}_{\mathrm{f}}=U \bar{X}^{\dagger} \bar{x}_{\mathrm{f}}$, where the last equality holds asymptotically for any choice of inputs satisfying the assumptions in Theorem 2.4

Remark 3: (Data-driven expressions with noisy data) Let the measurements of the input $u_{i}$ and the final state $x_{i}$ be corrupted by noise. Let $\tilde{U}=\left[u_{1}+w_{1} \cdots u_{N}+w_{N}\right]$ and $\tilde{X}=\left[x_{1}+v_{1} \cdots x_{N}+v_{N}\right]$ be the matrices obtained by concatenating all noisy measurements. The data-driven estimates (9), (11), and (18) computed from the noisy data $(\tilde{U}, \tilde{X})$ are typically biased. To see this, consider the system $x(t+1)=a x(t)+u(t), a \in \mathbb{R}, x_{0}=0$, and $T=N=1$. In this simple case, expressions (9), (11), and (18) are equivalent and, assuming that $x_{1}+v_{1} \neq 0$, read as $\hat{u}=\frac{u_{1}+w_{1}}{x_{1}+v_{1}} x_{\mathrm{f}}$. If $w_{1}$ and $v_{1}$ are independent random variables uniformly distributed in $[-\varepsilon, \varepsilon]$, with $0<\varepsilon<\left|u_{1}\right|$, it holds

$$
\begin{aligned}
\operatorname{Bias}[\hat{u}] & =\mathbb{E}_{w_{1}, v_{1}}[\hat{u}]-u^{*}=\mathbb{E}_{v_{1}}\left[\frac{u_{1}}{u_{1}+v_{1}}\right] x_{\mathrm{f}}-x_{\mathrm{f}} \\
& =\left[\frac{1}{2 \varepsilon} u_{1} \ln \left(\frac{u_{1}+\varepsilon}{u_{1}-\varepsilon}\right)-1\right] x_{\mathrm{f}},
\end{aligned}
$$

where $\mathbb{E}_{z}[\cdot]$ denotes the expected value with respect to $z$. It can be shown that, if $u_{1}$ and $x_{\mathrm{f}}$ are nonzero, the previous equation vanishes only in the limit $\varepsilon \rightarrow 0$. This implies that all datadriven expressions in this simple case are biased. When $n>1$, a quantitative characterization of the bias (and covariance) of the data-driven expressions appears to be difficult, due to the presence of pseudoinverse operations. However, numerical simulations with i.i.d. normally distributed noise (see also Fig. 2) suggest that (i) all data-driven expressions are biased in the case of noisy measurements, (ii) the magnitude of the bias is proportional to the standard deviation $\sigma$ of the noise for (11) and (18), while it increases rapidly as $\sigma$ grows and sets to a constant value for (9).

Remark 4: (Data-driven expressions with output measurements) Consider the system

$$
x(t+1)=A x(t)+B u(t), \quad y(t)=C x(t),
$$

where $C \in \mathbb{R}^{p \times n}$, and assume that for each experimental input $u_{i}, i \in\{1, \ldots, N\}$, we can measure the output of the system at time $T$, namely, $y_{i}=C x_{i}$. Let $Y=\left[\begin{array}{lll}y_{1} & \cdots & y_{N}\end{array}\right] \in \mathbb{R}^{p \times N}$

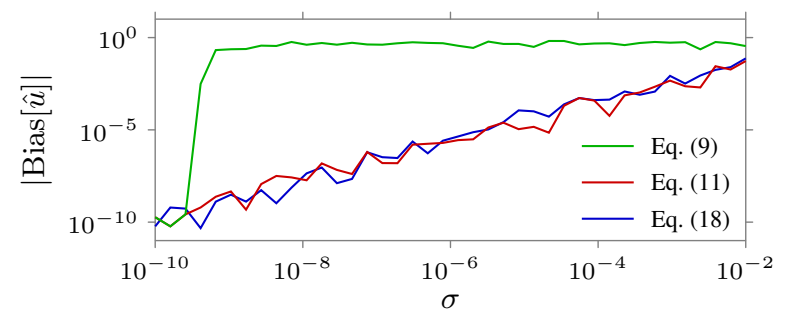

Fig. 2. This figure shows the magnitude of the bias of the data-driven expressions [9], [11, and [18] as a function of the standard deviation of the noise $\sigma$. We choose $A=\left[\begin{array}{ccc}-0.8 & 0 & 0 \\ 2 & 0.1 & 0 \\ 0.2 & 1 & 0.5\end{array}\right], B=\left[\begin{array}{l}1 \\ 0 \\ 0\end{array}\right], x_{\mathrm{f}}=\left[\begin{array}{c}0.3 \\ 1 \\ 0.5\end{array}\right], T=8$, and $N=10$. The entries of $U^{2}$ and $X$ have been chosen randomly and then corrupted by i.i.d. Gaussian noise with zero mean and standard deviation $\sigma$. The bias has been computed as the average over 100 noise realizations.

be the matrix concatenating all output measurements, and assume that the system is output controllable in $T$ steps. That is, the $T$-steps output controllability matrix $C_{O, T}=$ $\left[\begin{array}{lllll}C B & C A B & \cdots & C A^{T-1} B\end{array}\right]$ has full row rank [15]. The minimum-energy input to reach the output $y_{\mathrm{f}} \in \mathbb{R}^{p}$ in $T$ steps is $u^{*}=C_{O, T}^{\dagger}\left(y_{\mathrm{f}}-C A^{T} x_{0}\right)$. All results discussed in this paper apply to the case of output control after substituting $X$ and $x_{\mathrm{f}}$ with $Y$ and $y_{\mathrm{f}}$, respectively.

\section{NUMERICAL ANALYSIS}

What remains unclear from the previous analysis is the benefit, if any, in collecting a large number of control experiments. We next show that increasing the number of control experiments can improve the numerical reliability and accuracy of computing minimum-energy control inputs.

In Fig. 3 we compare the numerical performance of the model-based expressions of the minimum-energy controls $u^{*}=C_{T}^{\dagger} x_{\mathrm{f}}$ and $u^{*}=C_{T}^{\mathrm{T}} W_{T}^{\dagger} x_{\mathrm{f}}$ (Gramian-based), with our data-driven expressions in (9), (11), and (18). In particular, in Fig. 3(a)-(b) we plot the norm of the control inputs and the numerical errors in reaching the final state $x_{\mathrm{f}}$, for all strategies and as a function of the number $N$ of control experiments. Here, we focus on a "worst-case" analysis and choose a small input dimension $(m=2)$, since a large value of $m$ certainly improves the conditioning of all expressions. Fig. 3. (a) shows that the norm of the data-driven control inputs (9) and (11) equals its minimum value when $N \geq m T$ (as predicted by Theorems 2.1 and 2.3), whereas the norm of the data-driven input (18) converges to its minimum value only asymptotically (as predicted by Theorem 2.4. Fig. 3. (b) shows that, for sufficiently large $N$, the final state reached by the three data-driven control strategies is almost as close to $x_{\mathrm{f}}$ as the one computed via the model-based formula $u^{*}=C_{T}^{\dagger} x_{\mathrm{f}}$, and considerably closer to $x_{\mathrm{f}}$ than the state reached by the Gramian-based control input, with expressions (9) and (18) being the most accurate, showing that the computation of the minimum-energy control input via our data-driven expression is as reliable as the computation of the input based on the exact knowledge of the system matrices, and numerically more reliable than the model-based Gramian formula. Instead, in Fig. 3(c)-(d) we plot the norm of the control inputs obtained through the different strategies described above and their corresponding errors in the final state as a function of the system 


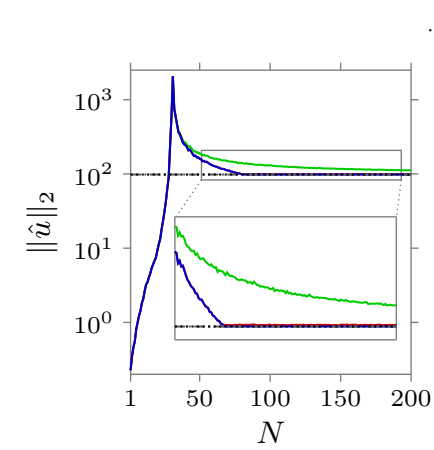

(a)

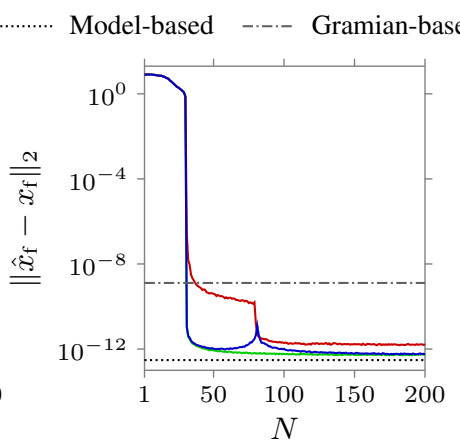

(b)

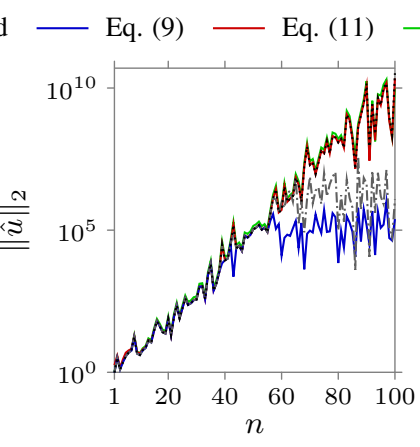

(c)

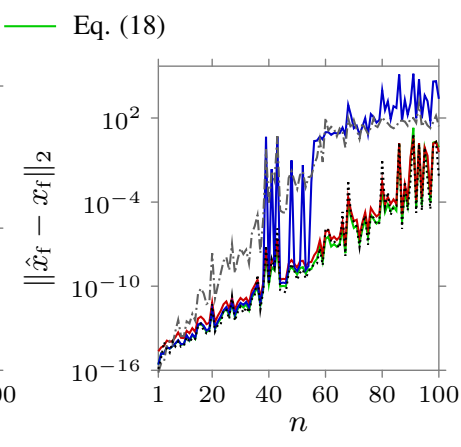

(d)

Fig. 3. Fig. 3 a)-(b) show the norm of the control input $\hat{u}$ computed via the model-based formula (5) (dotted line), via inversion of the controllability Gramian (dashed line), and via the data-driven expressions 9 , , 11), and (18) extended for $x_{0} \neq 0$ as in Remark 2 (colored lines), and the corresponding error in the final state, as the number of data $N$ varies. We choose $n=20, m=2$, and $T=40$. The matrix $A$ (possibly unstable) has been populated with random i.i.d. normal entries and then normalized by $\sqrt{n}$, the entries of $B, x_{0}$ and $x_{\mathrm{f}}$ have been chosen randomly according to a normal distribution. The curves represent the average over 100 experiments with data pairs $\left(x_{i}, u_{i}\right)$, where $u_{i}$ has random i.i.d. normal entries, and $x_{i}=C_{T} u_{i}$. Fig. 3 c)-(d) show the norm of the inputs $\hat{u}$ computed as above, and the corresponding errors in the final state, as a function of the system dimension $n$. We choose $m=2, T=n$, and $N=m T+20$. The matrices $A$ and $B$ have been generated as above. The curves represent the average over 1000 experiments with data $\left(x_{i}, u_{i}\right)$ and states $x_{0}, x_{\mathrm{f}}$ generated as above. All the computations have been carried out using standard built-in Mat $1 \mathrm{ab} 2016 \mathrm{~b}$ linear algebra routines.

dimension $n$. As expected, the accuracy of the Gramian-based control input deteriorates rapidly as $n$ increases. Yet, surprisingly, the data-driven expressions of the minimum-energy control inputs remain accurate for systems of considerably larger dimension. Further, the data-driven control (18) yields the smallest error in the final state among the three data-driven strategies. This could be due to the simpler form of (18), which requires the computation of only one pseudoinverse, or to the fact that the energy of (18) reaches the minimum value only asymptotically in $N$. Finally, Fig. 3.(c)-(d) show that expression (9) becomes numerically unreliable for smaller values of the system dimension compared to (11) and (18). This is likely because of the additional computations in (9).

\section{CONCLUSION AND FUTURE WORK}

In this paper we derive data-driven expressions of open-loop minimum-energy control inputs for linear systems. Leveraging linearity of the dynamics, we show that such optimal controls can be learned from a finite number of control experiments, without knowing or reconstructing the system matrices, and where the control experiments are conducted with non-optimal and arbitrary inputs. We derive three different data-driven expressions of minimum-energy controls: while (11) appears to be the simplest exact data-driven expression, (9) constitutes a radically different and new way of computing minimumenergy controls, and highlights several geometric connections between the minimum-energy solutions and the experimental data, and (18) provides a simple way of computing a family of data-driven, sub-optimal, minimum-energy controls. We further illustrate that our data-driven expressions of the minimumenergy inputs are simpler and numerically more reliable than the classic Gramian-based expression, especially when the dimension of the system increases.

The results of this paper support the intriguing idea of combining model-based control methods with data-driven techniques, showing that this new framework has the potential to considerably increase the reliability and effectiveness of the two parts alone. This paper also creates several directions of future research, including the extension to closed-loop, noisy, and model predictive control problems.

\section{REFERENCES}

[1] T. Kailath. Linear Systems. Prentice-Hall, 1980.

[2] A. Ben-Israel and T. N. E. Greville. Generalized inverses: theory and applications, volume 15 of CMS Books in Mathematics. Springer-Verlag New York, 2nd edition, 2003.

[3] S. Zhao and F. Pasqualetti. Networks with diagonal controllability gramians: Analysis, graphical conditions, and design algorithms. Automatica, 102:10-18, 2019.

[4] T. H. Summers, F. L. Cortesi, and J. Lygeros. On submodularity and controllability in complex dynamical networks. IEEE Transactions on Control of Network Systems, 3(1):91-101, 2016.

[5] F. Pasqualetti, S. Zampieri, and F. Bullo. Controllability metrics, limitations and algorithms for complex networks. IEEE Transactions on Control of Network Systems, 1(1):40-52, 2014.

[6] D. C. Sorensen and Y. Zhou. Bounds on eigenvalue decay rates and sensitivity of solutions to Lyapunov equations. Technical Report 02-07, Rice University, Houston, TX, 2002.

[7] J. Sun and A. E. Motter. Controllability transition and nonlocality in network control. Physical Review Letters, 110(20):208701, 2013.

[8] M. Gevers. Identification for control: From the early achievements to the revival of experiment design. European Journal of Control, 11:1-18, 2005.

[9] K. Furuta and M. Wongsaisuwan. Discrete-time LQG dynamic controller design using plant Markov parameters. Automatica, 31(9):1317-1324, 1995.

[10] G. Shi and R. E. Skelton. Markov data-based LQG control. Journal of Dynamic Systems, Measurement, and Control, 122(3):551-559, 2000.

[11] W. Aangenent, D. Kostic, B. de Jager, R. van de Molengraft, and M. Steinbuch. Data-based optimal control. pages 1460-1465, Portland, OR, USA, June 2005.

[12] G. R. Gonçalves da Silva, A. S. Bazanella, C. Lorenzini, and L. Campestrini. Data-driven LQR control design. IEEE Control Systems Letters, 3(1):180-185, 2019.

[13] C. De Persis and P. Tesi. On persistency of excitation and formulas for data-driven control. arXiv preprint arXiv:1903.06842, 2019.

[14] A. W. Van der Vaart. Asymptotic statistics, volume 3 of Cambridge Series in Statistical and Probabilistic Mathematics. Cambridge University Press, 2000.

[15] E. Kreindler and P. Sarachik. On the concepts of controllability and observability of linear systems. IEEE Transactions on Automatic Control, 9(2):129-136, 1964. 